\title{
Vigilância em saúde e território utilizado: possibilidades teóricas e metodológicas
}

\author{
Health surveillance and territory: \\ theoretical and methodological possibilities
}

Escola Politécnica de Saúde
Joaquim Venâncio,
Fundação Oswaldo Cruz,
Rio de Janeiro, Brasil.
2 Centro de Informação
Científica e Tecnológica,
Fundação Oswaldo Cruz,
Rio de Janeiro, Brasil.
Correspondência
M. Monken
Laboratório de Educação
Profissional em Vigilância
em Saúde, Escola Politécnica
de Saúde Joaquim Venâncio,
Fundação Oswaldo Cruz.
Av. Brasil 4036,
Rio de Janeiro, RJ
$21040-361$, Brasil.
mmonken@fiocruz.br

Abstract

Diagnoses of living conditions and health status, the constitutive elements for the reproduction of social life in various places, are listed and treated as contents disconnected from the territory. The recognition of social dynamics, habits, and customs is highly important for the determination of human health vulnerabilities, which originate in the interactions of social groups in given geographic spaces. The full use of the territory as a strategy for analyzing and intervening in health conditions presupposes the identification of geographic objects, their utilization by the population, and their importance for flows of persons and materials. It is thus necessary to develop methodologies for the recognition (both in the field and through secondary data) of objects and their forms, which are a condition for human action and existence. This article presents an approach to the incorporation of concepts from human geography in health practices, in light of two main authors: Milton Santos ("constitution of territory") and Anthony Giddens ("constitution of society").

Population Surveillance; Living Conditions; Geography
Maurício Monken 1

Christovam Barcellos 2

\section{Introdução}

A territorialização consiste em um dos pressupostos da organização dos processos de trabalho e das práticas de saúde, considerandose uma atuação em uma delimitação espacial previamente determinada. A territorialização de atividades de saúde vem sendo preconizada por diversas iniciativas no interior do Sistema Único de Saúde (SUS), como o Programa Saúde da Família, a Vigilância Ambiental em Saúde, Cidades Saudáveis e a própria descentralização das atividades de assistência e vigilância. No entanto, essa estratégia, muitas vezes, reduz o conceito de espaço, utilizado de uma forma meramente administrativa, para a gestão física dos serviços de saúde, negligenciando-se o potencial deste conceito para a identificação de problemas de saúde e de propostas de intervenção.

Muito além de ser meramente o espaço político-operativo do sistema de saúde, o território do distrito sanitário ou do município, onde se verifica a interação população-serviços no nível local, caracteriza-se por uma população específica, vivendo em tempo e espaço determinados, com problemas de saúde definidos e que interage com os gestores das distintas unidades prestadoras de serviços de saúde. Esse espaço apresenta, portanto, além de uma extensão geométrica, um perfil demográfico, epidemiológico, administrativo, tecnológico, polí- 
tico, social e cultural, que o caracteriza como um território em permanente construção 1 .

O reconhecimento desse território é um passo básico para a caracterização da população e de seus problemas de saúde, bem como para a avaliação do impacto dos serviços sobre os níveis de saúde dessa população. Além disso, permite o desenvolvimento de um vínculo entre os serviços de saúde e a população, mediante práticas de saúde orientadas por categorias de análise de cunho geográfico. Essa proposta, contida no novo modelo de vigilância em saúde, é justificada pelo agravamento das desigualdades sociais associado a uma segregação espacial aguda, que restringem o acesso da população a melhores condições de vida.

A concepção tradicional de saúde, pautada no modelo médico-assistencial, fez com que o setor saúde ficasse impotente em face dos problemas provocados pelo intenso processo de aglomeração e exclusão social 2. Dessa forma, vem se fortalecendo a idéia das ações de promoção da saúde, orientadas para as ações coletivas e intersetoriais, independentemente do sistema de atenção à saúde. A atenção voltada para a produção social da saúde das populações gera a necessidade de esclarecer as mediações que operam entre as condições reais em que ocorre a reprodução dos grupos sociais no espaço e a produção da saúde e da doença.

Neste trabalho, é destacado o papel do território utilizado pelas populações na compreensão das situações de saúde, utilização que se dá em face de diferentes contextualidades 3 , entendidas por meio da análise processual das práticas sociais cotidianas. O objetivo principal desta pesquisa é contribuir para a construção de metodologias de reconhecimento do território, voltadas para a vigilância em saúde, mediante a incorporação de determinadas categorias geográficas. Posteriormente, por intermédio da abordagem teórica de dois autores principais, Milton Santos e Anthony Giddens, são relacionados conceitos e categorias do processo de territorialização para a organização e instrumentalização de práticas de vigilância em saúde nos serviços.

\section{Práticas de vigilância em saúde e a territorialização}

Os problemas de saúde apresentam uma diversidade de determinações, fazendo com que propostas de resolução sejam baseadas em múlti- plas estratégias, medidas e atores. Destaca-se, ainda, nesse processo, o conhecimento popular e a participação social decorrente desse saber como base para a formulação conceitual e das ações de promoção da saúde.

Para Mendes 1, a reorientação dos sistemas de saúde na direção de afirmar-se como "espaço da saúde”, e não exclusivamente da atenção à doença, exige um processo de construção social de mudanças que se darão, concomitante e dialeticamente, na concepção do processo saúde-doença, no paradigma sanitário e na prática sanitária. O entendimento do processo saúde-doença tem evoluído consideravelmente de uma concepção eminentemente monocausal do pensamento clínico a concepções ampliadas de saúde, que articulam saúde com condições de vida, o que a Carta de Ottawa e todo o movimento contemporâneo da promoção social da saúde incorporaram plenamente 4,5 . Essa nova visão considera a saúde como uma acumulação social, expressa num estado de bemestar, que pode indicar acúmulos positivos ou negativos. Portanto, compreende que a dinâmica das relações sociais seja o fator que define as necessidades de cuidados à saúde.

Para o setor saúde, a perspectiva do modelo da vigilância em saúde configura-se no constructo operacional que se propõe a dar resposta aos problemas de saúde. Castellanos 6 aponta que o entendimento dos problemas que estruturam uma situação de saúde parte do ponto de vista do ator social que a descreve e explica. $\mathrm{O}$ autor propõe que os fenômenos de saúde e doença ocorrem em diferentes dimensões: as singulares, entre indivíduos ou entre agrupamentos de população por atributos individuais; as particulares, isto é, entre grupos sociais em uma mesma sociedade, e as gerais, que são os fluxos e fatos que correspondem à sociedade em geral. A definição de problema de saúde e o seu potencial de transformação são diretamente correspondentes a cada uma dessas dimensões.

Especialmente interessante para a vigilância em saúde são os problemas definidos na dimensão conceituada como particular, pois, nesse nível, os problemas emergem como características de grupos de população, em conjunto com seus processos de reprodução social, configurando-se em comunidades, ou "grupos sócio-espaciais particulares" 7. Não por acaso, a noção usual de comunidade envolve citações de palavras-chave como lugar, laços sociais e ação ${ }^{8}$. As ações e práticas que derivam dessa abordagem particular permitem trabalhar em 
períodos mais precoces do processo de determinação e, ao mesmo tempo, ampliar as estratégias de atenção primária como um conjunto de ações sociais dirigidas a essas comunidades para a promoção da qualidade de vida.

Para a constituição de uma base organizativa dos processos de trabalho nos sistemas locais de saúde em direção a essa nova prática, é importante o reconhecimento dos territórios e seus contextos de uso, uma vez que estes materializam diferentemente as interações humanas, os problemas de saúde e as ações sustentadas na intersetorialidade. Cabe à vigilância em saúde exercer o papel organizativo dos processos de trabalho em saúde mediante operações intersetoriais, articuladas por diferentes ações de intervenção (promoção, prevenção, atenção), fincada em seus três pilares estratégicos: os problemas de saúde, o território e a prática intersetorial 9. Diante disso, o conceito de espaço, de onde se origina a noção de território, pode exercer importante papel na organização das práticas de vigilância em saúde.

A vigilância em saúde tem sido compreendida de três formas: como análise e monitoramento de situações de saúde; como integração institucional entre atividades de vigilância epidemiológica e sanitária; como elemento que pressupõe a organização tecnológica do trabalho de redefinição das práticas sanitárias 9. Essa redefinição está voltada para o planejamento, seja numa dimensão técnica, ao conceber a "vigilância da saúde" como um modelo assistencial alternativo, que combina tecnologias distintas, destinadas a controlar determinantes, riscos e danos 10, seja numa dimensão gerencial, que organiza os processos de trabalho em saúde sob a forma de operações, para confrontar problemas num território delimitado 1 . A “vigilância da saúde" é entendida como "uma dada organização tecnológica do trabalho" 10 , que atua produzindo práticas sob a forma de operações que se estruturam de acordo com as diferentes fases ou dimensões do processo saúde-doença, desde os agravos a situações de exposição, às necessidades sociais de saúde 1 .

\section{A natureza do território, a dimensão local e o cotidiano}

A análise do território serve, antes de tudo, como meio operacional para avaliação objetiva das condições criadas para a produção, circulação, residência, comunicação e sua relação com as condições de vida ${ }^{11}$. Além disso, esse território é um meio percebido, subordinado a uma avaliação subjetiva de acordo com repre- sentações sociais específicas. O seu entendimento é ainda impreciso e abordado de acordo com diversos pontos de vista, estando não só associado a uma porção específica da Terra, identificada pela natureza, pelas marcas que a sociedade ali imprime, como também a uma simples localização, referida indiscriminadamente a diferentes escalas, como a global, regional, da cidade, da rua e até de uma casa apenas. O espaço geográfico é definido por Santos 12 (p. 51) como um "conjunto indissociável de sistemas de ações e objetos”. Para que adquiram materialidade, esses objetos, tanto naturais, quanto elaborados tecnicamente, e, ainda, os eventos da vida precisam estar situados no espaço e no tempo. Para Santos 12 (p. 52), os objetos "são esse extenso, essa objetividade, isso que se cria fora do indivíduo e se torna instrumental de sua vida, tal uma cidade, barragem, estradas de rodagem, portos, etc. São do domínio tanto da Geografia Física quanto da Geografia Humana que, através da história desses objetos, da forma como foram produzidos e mudam, essas geografias se encontram".

Com a técnica - conceito-chave da obra de Santos 12 -, o indivíduo em sociedade forma um conjunto de meios instrumentais e sociais com os quais realiza sua vida, produz e, ao mesmo tempo, cria espaço. Essa concepção de espaço leva em conta todos os objetos existentes numa extensão contínua, supondo a coexistência desses objetos como sistemas e não apenas como coleções: a utilidade atual dos objetos, passada ou futura, vem exatamente do seu uso combinado pelos grupos humanos que os criaram ou que os herdaram das gerações anteriores. Seu papel, porém, além de funcional, é também simbólico. Desse modo, a identificação desses objetos, seus usos pela população e sua importância para os fluxos das pessoas e de materialidades são de grande relevância para o reconhecimento da dinâmica social, hábitos e costumes, bem como na determinação de vulnerabilidades para a saúde humana, originadas nas interações de grupos humanos em determinados espaços geográficos.

A racionalidade do espaço, entendida historicamente e fruto das redes, é expressa por meio do "conteúdo geográfico do cotidiano" 13. Esse conceito pode contribuir para desvendar a (re)produção do sistema através de sua obviedade e concretude. A globalização fez redescobrir a corporeidade, revelada como uma certeza materialmente sensível, em virtude da fluidez, velocidade e referência a lugares e coisas distantes 14. Esse processo fez reaparecer, no cenário científico, a dimensão local, aproximando os verdadeiros significados da reali- 
dade social através da consideração do cotidiano.

A característica mais importante do lugar é, antes de tudo, de natureza interna, cuja extensão confunde-se com sua própria existência, tendo uma configuração física, ou melhor, territorial. Essa característica fundam a escala do cotidiano e seus parâmetros são a co-presença, a vizinhança, a intimidade, a emoção, a cooperação e a socialização com base na contigüidade, reunindo na mesma lógica interna todos os seus elementos: pessoas, empresas, instituições, formas sociais e jurídicas e formas geográficas. O cotidiano imediato, localmente vivido, traço de união de todos esses dados, é a garantia da comunicação 13. Assim, a análise da "dimensão espacial do cotidiano" 13 permite, sobretudo, concretizar as ações e as práticas sociais, conduzindo ao entendimento diferenciado dos usos do território, das ações e as formas geográficas que podem formar contextos vulneráveis para a saúde.

\section{Aproximação metodológica do território utilizado e a situação de saúde}

A categoria de análise fundamental para a territorialização em vigilância em saúde é a de "território utilizado" 15 , que supera o antigo problema de análise para o entendimento da interação pessoa-mundo, considerando-se o sentido da "interdependência e a inseparabilidade entre a materialidade e o seu uso, o que inclui a ação humana" 15 (p. 247). Conforme os autores, a categoria território somente pode ser usada mediante o reconhecimento dos atores que dele se utilizam. Os usos se diferenciam conforme os períodos históricos, fazendo com que se busque a "evolução dos contextos e, assim, as variáveis trabalhadas no interior de uma situação" 15. Para se discutir o território utilizado, deve-se analisar a "constituição do território" 13 , que consiste numa proposta para uma geografia eminentemente empiricista. " $O$ mundo das coisas, das ações e das relações é perceptível, ao menos tendencialmente, em todos os lugares (...) e o processo da construção da teoria pode fundar-se, então, muito mais no empírico, no realmente existente" 15 (p. 20). Esta nova situação histórica é chamada de "produção da universalidade empírica” 15 (p. 20).

Essas relações sociais são projetadas no espaço e são menos duradouras que o espaço em si 16. Por intermédio da categoria território utilizado, o planejamento da vigilância em saúde pode ampliar seu campo de atuação formal so- bre o espaço, que, por sua vez, modifica-se conforme a dinâmica das relações sociais. Assim, a escala geográfica operativa para a territorialização emerge, principalmente, dos espaços da vida cotidiana, compreendendo desde o domicílio (dos programas de saúde da família) a áreas de abrangência (de unidades de saúde) e territórios comunitários (dos distritos sanitários e municípios). Esses territórios abrangem, por isso, um conjunto indissociável de objetos cujos conteúdos são usados como recursos para a produção, habitação, circulação, cultura, associação e lazer.

Segundo Giddens 3, o reconhecimento das fontes de cerceamento da atividade humana, produzidas pela natureza do próprio corpo humano e pelos espaços físicos em que a atividade ocorre, permite identificar os limites para o comportamento das pessoas nos territórios de vida. Ao se observarem as rotinas descritas, são reconhecidas as formas geográficas cujos conteúdos lhes dão existência concreta. Os padrões típicos dos movimentos das pessoas, em outras palavras, podem ser representados como a repetição de atividades de rotina ao longo dos dias ou de períodos mais longos. As pessoas movimentam-se em espaços físicos cujas propriedades interagem com suas capacidades, dadas as restrições apontadas por suas fronteiras físicas, sociais e simbólicas. Portanto, na maior parte dos dias, a mobilidade se dá dentro de áreas restritas. A conduta da vida cotidiana de um indivíduo promove a apreensão sucessiva de características dos territórios (cenários de interação, segundo Giddens 3 ), tais como outras pessoas, objetos dos territórios do cotidiano e materialidades, como o ar, água e alimentos.

O termo lugar deve, assim, ser associado não só à localização no espaço, mas à idéia de presença, explicado tanto pela sua espacialidade, quanto pela sua temporalidade, ou seja, da mutualidade da presença do corpo e da "ausência presente" contida nas instituições da vida social (tanto formais quanto informais). As propriedades do lugar são usadas permanentemente pelas pessoas na constituição dos encontros através do espaço e do tempo. É nos lugares onde se dá a interseção das atividades de rotina de diferentes pessoas, que as características do espaço são usadas rotineiramente para constituir o conteúdo significativo da interação de pessoas na vida social 3 . A rede territorial de conexões dessas interações humanas, ao se intensificar coletivamente, revela-se em fluxos, nos quais uma certa tipologia se institucionaliza, ditando regras, comportamentos e funções. Cabe ao espaço interagir e acolher usos característicos a esses fluxos e interações. 
A proposta de identificação dos territórios de vida dos grupos sociais e suas práticas cotidianas deve ter como ponto de partida o mapeamento dos percursos e fluxos diários, interações e a malha de redes microgeográficas, que serão úteis para trabalhar uma "epidemiologia geográfica do cotidiano”, entendida com base nas necessidades e nos problemas de saúde de populações. Alguns elementos de dimensão espacial devem ser destacados nessa abordagem. Os objetos (fixos) e as ações (fluxos) no espaço produzem elementos espaciais básicos para a vida cotidiana, que realizam o diálogo da pessoa com o mundo, estabelecendo com isso uma "conexão materialística" 13 de uma pessoa com a outra. Estruturam-se, assim, as seguintes “dimensões espaciais do cotidiano" 13 (p. 257):

- os percursos 17 podem ser objetos geográficos que propiciam as ações e os seus diversos fluxos de matéria e pessoas, como as estradas, vias de pedestres, linhas de transportes públicos, canais de navegação, ferrovias, ruas e becos;

- por barreiras físicas ou margens 17 deve-se entender interrupções lineares de continuidades, ou fronteiras físicas dos objetos, não utilizadas como percursos pelos indivíduos e grupos sociais, mas que canalizam as ações num sentido ou outro. Por exemplo, margens de rios, de grandes avenidas, margens de desenvolvimento de construções, de prédios, muros e rugosidades naturais diversas do terreno;

- por nós 14 entendem-se os pontos em direção aos quais e a partir dos quais o indivíduo se movimenta: agem em fase de concentração uma praça, a esquina - e de conjunção - os cruzamentos das estradas, os portos e aeroportos, os caminhos etc. A idéia de nó está intimamente relacionada à de percurso, podendo também ser compreendido como ponto ou estação, onde as interações sociais convergem para objetos (fixos) que detêm uma determinada tipologia de ações (fluxos);

- a idéia de estação ${ }^{18,}$ portanto, ajuda no entendimento proposto; é o lugar ou ponto de parada onde a mobilidade física das trajetórias dos agentes e de materialidades é suspensa ou reduzida nos encontros ou nas ocasiões sociais. O lugar em que se estabelece o nó é onde acontece a interseção de atividades de diferentes indivíduos. Pode ser um espaço para a produção, o comércio ou para os serviços, o exercício do lazer, da cultura e da religião, ou de associativismos diferenciados.

Nesses movimentos, as características dos cenários, segundo Giddens 3 , e do conjunto de objetos, conforme Santos 12, são usadas rotineiramente para dar o conteúdo à interação. A concentração da vida social em determinados locais está intimamente ligada a uma especificidade do ser social, ou seja, aglomera ou acumula as suas atividades em torno de centros ou nós geográficos passíveis de serem identificados. Essas dimensões espaciais ordenadoras, e ao mesmo tempo ordenadas, pelo uso dado ao território, estruturam materialmente o espaço através dos objetos e ações que configuram contextualidades 3 , cujas regras e recursos para utilização do território são apropriados pela população nas práticas sociais da vida cotidiana.

A identificação de problemas de saúde no território deve, conseqüentemente, suplantar a listagem de agravos prevalentes e evidenciáveis, mediante notificações, para abordar e contemplar a compreensão das vulnerabilidades e dos determinantes. O ponto de partida desse processo é a territorialização do sistema de saúde, isto é, o reconhecimento e o esquadrinhamento do território do município, segundo a lógica de relações entre condições de vida, saúde e acesso às ações e serviços de saúde, o que implica um processo de coleta e sistematização de dados demográficos, sócio-econômicos, político-culturais, epidemiológicos e sanitários 9. Nesse sentido, as técnicas de geoprocessamento têm auxiliado na organização e análise espacial de dados sobre ambiente, sociedade e saúde, permitindo a elaboração de diagnósticos de situação e o intercâmbio de informações entre setores (ver, por exemplo, o número temático de Cadernos de Saúde Pública sobre análise de dados espaciais em saúde 19). Esse conjunto de técnicas vem sendo gradativamente incorporado à prática de vigilância em saúde 20 , e, paralelamente, observa-se um intenso debate no Brasil sobre a incorporação do conceito de espaço geográfico no campo da saúde coletiva 21 . Deve-se, nesse caso, avaliar as propostas metodológicas e os conceitos de espaço geográfico subjacentes a essas técnicas 22.

Freqüentemente, nos diagnósticos de condições de vida e de situação de saúde, os elementos constitutivos da reprodução da vida social nos diversos lugares são listados e tratados como conteúdos desarticulados do território analisado. Uma proposta de vigilância em saúde baseada no território deve, também, considerar os sistemas de objetos naturais e construídos, identificando seus diversos tipos de ações, a forma como são percebidos pela população, o papel das regras de utilização dos recursos para promover determinados hábitos e comportamentos, bem como problemas de saúde cujas características são passíveis de identificação.

Para (re)conhecer os usos diversificados do território, é necessário investigar as práticas 
sociais transformadas em rotina no espaço. Uma das propostas deste trabalho é conjugar o estudo da "constituição do território", já apontada por Milton Santos 23, à teoria da estruturação de "constituição da sociedade", do sociólogo inglês Anthony Giddens 3, para compreender os contextos de utilização do território por parte das populações.

A teoria da estruturação de Giddens 3 é uma tentativa de formular uma descrição plausível da atividade humana e de sua estrutura. Giddens assinala que a base de sua teoria não é privilegiar o ator individual e nem a existência de qualquer forma de totalidade social, mas lançar os olhos sobre as práticas sociais que são ordenadas no tempo e no espaço. Essa ordenação se dá por meio da integração social, em circunstâncias de co-presença ou de conexões com aqueles que estão fisicamente ausentes. Um exemplo são os processos de trabalho instituídos por organizações sociais. Seu mecanismo pressupõe a integração social, que é distinta da que está envolvida em contexto de copresença, e uma organização espacial particular 22. A integração sistêmica traz em si as questões das instituições sociais, normatizadas tanto formalmente, como informalmente. É a natureza da interação que caracteriza e explica a conduta social nos contextos. As contextualidades são lugares dotados de bidimensionalidade, reconhecíveis à medida que neles são difundidas características particulares, nos quais os indivíduos e os grupos sociais atuam. Em outros termos, é o que atribui um significado singular ao lugar, como resultado de uma rede de ações construídas historicamente 24 .

Dessa forma, entende-se que os lugares e sua constituição territorial tornam-se vitais para assegurar a fixidez subjacente às instituições. A conduta humana nesses contextos é reproduzida mediante atividades humanas recursivas, continuamente recriadas pelas pessoas através dos próprios meios pelos quais elas se expressam. Em outras palavras, a vida social ostenta uma repetitividade essencial, passível, assim, de aproximações metodológicas para seu entendimento.

As interações, por sua vez, envolvem a reprodução de práticas sociais de pessoas e seus encontros, sendo as regras e recursos implicados nessa reprodução essenciais para a manutenção da vida social. Mantêm, no entanto, uma repetitividade criadora, em que os contextos de interação social nunca são exatamente os mesmos; são únicos, singulares. Essa estrutura sócio-espacial pode ter pequena extensão, na qual determinadas regras se manifestam pela interação entre pessoas em situações de co- presença, ou grande extensão, em que a expressão de outras regras não depende da co-presença, mas das instituições. Entretanto, as regras não podem ser conceituadas separadamente dos recursos, pois estes se constituem nos meios necessários para a realização material das ações e práticas sociais.

A estruturação da interação humana, que interessa particularmente para as análises de situação de saúde, implica reciprocidade das práticas baseadas nas regras e recursos (de autonomia e dependência) entre pessoas e coletividades. Portanto, o conceito de contexto de uso do território orienta a articulação teórica entre as categorias que possibilitam compor analiticamente a "constituição do território", de Milton Santos 23, e "da sociedade", de Anthony Giddens 3, propostas na Figura 1.

Os recursos implicados na reprodução da vida social podem ter uso comum, pelo coletivo social no território, estabelecido nos fluxos de pessoas e de matéria, tais como os equipamentos urbanos. Os recursos individuais são relativos às condições do domicílio e às instalações sanitárias da habitação. O nível de renda materializa-se nessas condições do domicílio e na posse de equipamentos domésticos que, por sua vez, podem condicionar regras específicas de comportamento.

Os recursos coletivos compreendem um “conjunto dos sistemas naturais, herdados por uma determinada sociedade e os sistemas de engenharia, isto é, objetos técnicos e culturais historicamente estabelecidos" 23 (p. 26), que são apenas condições. Sua significação real advém das ações realizadas sobre elas. Esses objetos estabelecem uma conexão entre pessoas, promovendo ou limitando essas ações. Implicam não só percursos, barreiras, como também estações de encontro. Sendo assim, os objetos geográficos relevantes para a vigilância em saúde são: a infra-estrutura de ocupação do lugar (estradas e ruas, caminhos, sistemas de esgoto e de água, depósitos de lixo, núcleos habitacionais, novos assentamentos e invasões) e suas condições ecológicas e geomorfológicas (áreas florestadas e desmatadas, fauna e flora, relevo, hidrografia).

Os agrupamentos populacionais podem apresentar contextos de uso de recursos que condicionam, muitas vezes, determinados comportamentos. O território socialmente utilizado adquire características locais próprias, e a posse de determinados recursos expressa a diferenciação de acesso aos resultados da produção coletiva, da sociedade. Albuquerque 25 ressalta uma associação que baseia o uso de categorias geográficas para a vigilância em saúde. 


\begin{tabular}{|c|c|c|c|}
\cline { 2 - 3 } \multicolumn{1}{c|}{} & Regras & Recursos & \multicolumn{2}{c|}{$\begin{array}{c}\text { Constituição } \\
\text { do território }\end{array}$} \\
\hline Sistemas de objetos & & & \\
\hline \multirow{2}{*}{ Sistemas de ações } & & & \\
\hline \multicolumn{2}{|c|}{ Constituição da sociedade } & \\
\cline { 2 - 3 } & & &
\end{tabular}

Para a autora, a constituição do território "refletiria as posições ocupadas pelas pessoas na sociedade e conseqüência de uma construção histórica e social, sendo, por isso, capaz de refletir as desigualdades existentes" 25 (p. 613). Indica, com isso, que os "hábitos e comportamentos considerados como fatores causais ou protetores para essas doenças ou eventos, tais como fumo, alimentação, agentes tóxicos, uso de preservativos etc, parecem circular de forma diferenciada em grupos populacionais" 25 (p. 613). A essa abordagem soma-se a preocupação crescente em distinguir níveis e perfis de risco decorrentes das desigualdades sociais 26 .

O reconhecimento do território na escala do cotidiano não exclui a identificação de relações de verticalidade com outros níveis de decisão que podem influenciar sobremaneira a vida social local. Importante exemplo dessa influência traduz-se nos efeitos da presença de firmas multinacionais. A ação global exercida por firmas globais escolhe frações do mundo sobre as quais deseja atuar e as fragmenta ainda mais. Esses efeitos podem trazer inúmeros problemas de saúde, tanto diretos, pela emissão de poluentes, quanto indiretos, em virtude da ação desestruturadora de sua inserção local. Por outro lado, a partir da localização territorial de problemas de saúde, pode-se apreender o feixe de relações que caracterizam a situação-problema. Extrapolando as escalas territoriais de trabalho da vigilância em saúde, por intermédio da noção de horizontalidade, pode-se situar espacialmente o problema de saúde e analisar as influências, seja no seu entorno, seja no seu contexto mais amplo.

\section{Considerações finais}

Ao longo do desenvolvimento científico, a geografia e a epidemiologia têm intercambiado conceitos e paradigmas, o que tem provocado mudanças de método e do próprio foco de atenção de pesquisas. A evolução da epidemiologia foi sintetizada por Susser \& Susser 27, que destacam três períodos com métodos e concepções teóricas diferentes: as estatísticas sanitárias e a teoria miasmática; a epidemiologia das doenças infecciosas e a teoria dos germes; finalmente, a epidemiologia das doenças crônicas e a teoria da multicausalidade. Esses mesmos autores apontam a necessidade de desenvolvimento de uma "eco-epidemiologia”, que dê conta dos múltiplos níveis em que os problemas de saúde se manifestam, desde as vulnerabilidades individuais até os macrodeterminantes sociais e ecológicos.

Da mesma maneira que cada um desses períodos produziu metodologias para a análise de condições de saúde, estruturou um modo de pensar e agir no campo. Assim, a vigilância em saúde pautou-se, no primeiro período, nos levantamentos ambientais, que explicariam o “contágio", a produção local de doenças, e permitiriam intervir sobre características do terreno que as gerou. No segundo período, o trabalho no campo foi voltado para a identificação da "história natural das doenças", os vetores, microorganismos, clima e hospedeiros, que, formando ciclos de contaminação-transmissão, produziriam doenças. No terceiro período, o trabalho de campo é marcado pela busca de "fatores de risco", que explicariam a produção de doenças. Este último paradigma produziu a maior parte dos roteiros utilizados atualmente para a prática de vigilância em saúde, como os questionários de investigação epidemiológica, voltados para a argüição do doente sobre possíveis formas de exposição. Esse enfoque traz importantes vieses para a compreensão do processo de saúde-doença. Em primeiro lugar, procura causas da doença na sua própria vítima, reforçando estigmas para indivíduos enfermos 
28. Em segundo lugar, o resgate das condições sócio-ambientais que promoveram a doença é realizado procurando reaver o contato do indivíduo já doente com outros indivíduos e o ambiente, o que certamente não representa a complexidade das relações sociais existentes em uma comunidade.

A busca de novos paradigmas para o campo da saúde coletiva deve ser acompanhada pelo desenvolvimento de métodos que articulem os níveis do indivíduo e das coletividades, vistas não como um agregado de pessoas, mas como um todo, com características particulares, organização própria e território. Dessa forma, a vigilância em saúde carece de instrumentos que incorporem a dimensão do lugar, como expressão do relacionamento entre grupos sociais e seu território. A compreensão do conteúdo geográfico do cotidiano na dimensão local tem grande potencial não só explicativo, como também de identificação de situaçõesproblema para a saúde e, com base nisso, de planejamento e de organização das ações e práticas de saúde nos serviços. A análise sistêmica do contexto local, em escalas geográficas do cotidiano como as apresentadas no trabalho, permite identificar a formação contextual de uma situação de saúde, no espaço e no tempo, podendo ser de grande utilidade para a vigilância em saúde.

\section{Resumo}

Nos diagnósticos de condições de vida e de situação de saúde, os elementos constitutivos da reprodução da vida social nos diversos lugares são listados e tratados como conteúdos desarticulados do território. O reconhecimento da dinâmica social, hábitos e costumes é de grande importância para a determinação de vulnerabilidades para a saúde humana, originadas nas interações de grupos sociais em determinados espaços geográficos. O uso pleno do território como estratégia de análise sobre condições de saúde e intervenção nestas pressupõe a identificação de objetos geográficos, sua utilização pela população e sua importância para os fluxos das pessoas e materiais. Para isso, é necessário o desenvolvimento de metodologias para o reconhecimento, em campo e mediante dados secundários, de objetos e suas formas, que são condições da ação $e$ meios de existência do agir humano. Neste trabalho, é apresentada uma aproximação para a incorporação de conceitos da geografia humana nas práticas de saúde, à luz de dois autores principais: Milton Santos ("constituição do território") e Anthony Giddens ("constituição da sociedade”).

Vigilância da População; Condições de Vida; Geografia

\section{Colaboradores}

A revisão de conceitos de geografia e de saúde coletiva e a redação do artigo foram realizadas por ambos os autores. 


\section{Referências}

1. Mendes EV. Distritos sanitários: processo social de mudanças nas práticas sanitárias para Sistema Único de Saúde. São Paulo: Editora Hucitec/Rio de Janeiro: ABRASCO; 1993.

2. Waltner-Toews D. The end of medicine: the beginning of health. Futures 2000; 32:655-7.

3. Giddens A. A constituição da sociedade. São Paulo: Martins Fontes; 1989.

4. Buss PM, Ferreira JR. Promoção da saúde e a saúde pública: contribuição para o debate entre as escolas de saúde pública da América Latina. Rio de Janeiro: Escola Nacional de Saúde Pública, Fundação Oswaldo Cruz; 1998.

5. Ferraz ST. Cidades Saudáveis - uma urbanidade para 2000. Brasília: Paralelo 15; 1999.

6. Castellanos PL. On the concept of health and disease. Description and explanation of the health situation. Epidemiol Bull 1990; 10:1-8.

7. Sabroza PC, Leal MC. Saúde, ambiente e desenvolvimento: alguns conceitos fundamentais. In: Leal MC, Sabroza PC, Rodriguez RH, Buss PM, organizadores. Saúde, ambiente e desenvolvimento. vol. 1. São Paulo: Editora Hucitec/Rio de Janeiro: ABRASCO; 1992. p. 45-93.

8. MacQueen KM, McLellan EMA, Metzger DS, Kegeles S, Strauss RP, Scotti RMA, et al. What is community? An evidence-based definition for participatory public health. Am J Public Health 2001; 91:1929-36.

9. Teixeira CF, Paim JS, Villasbôas AL. SUS, modelos assistenciais e vigilância da saúde. Inf Epidemiol SUS 1998; 7:7-28

10. Paim JS. A reforma sanitária e os modelos assistenciais. In: Rouquayrol MZ, organizador. Epidemiologia e saúde. São Paulo: Medsi; 1994. p. 45566.

11. Unglert CVS. Territorialização em sistemas de saúde. In: Mendes EV, organizador. Distritos sanitários: processo social de mudanças nas práticas sanitárias para o Sistema Único de Saúde. São Paulo: Editora Hucitec/Rio de Janeiro: ABRASCO; 1993. p. 221-35.

12. Santos M. Metamorfoses do espaço habitado. São Paulo: Editora Hucitec; 1988.

13. Santos M. A natureza do espaço: técnica e tempo, razão e emoção. São Paulo: Editora Hucitec; 1996.

14. Soja E. Geografias pós-modernas: a reafirmação do espaço na teoria social crítica. São Paulo: Jorge Zahar Editor; 1993

15. Santos M, Silveira ML. O Brasil: território e sociedade no início do século XXI. Rio de Janeiro: Record; 2001.
16. Souza MJL. Urbanização e desenvolvimento no Brasil atual. São Paulo: Ática; 1996.

17. Bettanini T. Espaço e ciências humanas. Rio de Janeiro: Paz e Terra; 1982.

18. Hägerstrand $T$. What about people in regional science? Papers of the Regional Science Association 1970; 24:7-21

19. Carvalho MS, Nobre FF [Editorial]. Cad Saúde Pública 2001; 17:1056-7.

20. Rojas LI, Barcellos C, Peiter P. Utilização de mapas no campo da epidemiologia no Brasil: reflexões sobre trabalhos apresentados no IV Congresso Brasileiro de Epidemiologia. Inf Epidemiol SUS 1999; 8:25-35.

21. Czeresnia D, Ribeiro AM. O conceito de espaço em epidemiologia: uma abordagem histórica e epistemológica. Cad Saúde Pública 2000; 16:595605.

22. Barcellos C, Machado JHM. A organização espacial condiciona as relações entre ambiente e saúde: o exemplo da exposição ao mercúrio em uma fábrica de lâmpadas fluorescentes. Ciênc Saúde Coletiva 1998; 3:103-13.

23. Seabra CM, Leite JC. Território e sociedade: entrevista com Milton Santos. São Paulo: Editora Fundação Perseu Abramo; 2000.

24. Randolph R. A contextualidade de locais e a totalidade social: uma interpretação das práticas de quatro grupos sociais rurais e urbanos. In: Souza MA, Santos M, Scarlato F, Arroyo M, organizadores. O novo mapa do mundo. São Paulo: Editora Hucitec; 1992. p. 128-32.

25. Albuquerque MFM. Debate sobre o artigo de Czeresnia \& Ribeiro. Cad Saúde Pública 2001; 16:612-3.

26. Barata RB. Condições de vida e situação de saúde. Rio de Janeiro: ABRASCO; 1997.

27. Susser M, Susser E. Choosing a future for epidemiology. I. Eras and paradigms. Am J Public Health 1996; 86:668-73.

28. Valla VV. Revendo o debate em torno da participação popular: ampliando sua concepção em uma nova conjuntura. In: Barata, RB, Briceño-Leon R, organizadores. Doenças endêmicas: abordagens sociais, culturais e comportamentais. Rio de Janeiro: Editora Fiocruz; 2000. p. 251-68.

Recebido em 06/Mai/2004

Versão final reapresentada em 25/Ago/2004

Aprovado em 20/Set/2004 